\title{
Security that Matters: General Knowledge and Correlations in the Context of Applied Satellite Navigation, Specific Interference-Events and the Use of the GNSS-Technology
}

Janika Saretzki

ERC Experience Research \& Consulting, Austria

Jürgen Pretsch

ERC Experience Research \& Consulting, Austria

Günther Obertaxer

Joanneum Research - Digital, Austria

Roman Lesjak

Joanneum Research - Digital, Austria

\section{Abstract}

Navigation through global navigation satellite systems (GNSS) has become an indispensable part of modern life with threats such as GNSS interference, making it necessary to uncover relevant psychological aspects in the context of the GNSS construct, diverse interference events, and the use of related technologies. A total of $n=122$ subjects participated in an online survey, which included scales and specifically constructed items on GNSS usage, acceptance, dependence, and self-assessed sense of direction and relevance of basic psychological needs. In addition, frequently emphasized factors influencing acceptance and use of diverse technologies were recorded according to the Unified Theory of Acceptance and Use of Technology (UTAUT; Venkatesh et al., 2003). Correlation analyses showed that the frequency of GNSS use was associated with both effort expectation of appropriate technologies, hedonistic motivation, habits of using GNSSenabled devices, and specific aspects of mobility. In terms of reported GNSS dependency, negative correlations were found with self-assessed orientation ability. It was also possible to identify voluntariness in the use of related technologies, the age of the users, and the relevance of self-determination as essential variables in the context of GNSS use. The results underline the need for further investigation of psychological aspects and contribute to existing discussions in the context of various threat scenarios.

Keywords: GNSS, GPS, Security Research, GNSS Interferences, Jamming, Spoofing 


\section{Introduction}

The usage of earth satellites for navigation purposes represents a technology that has been in use for more than 50 years (e.g., Dovis, 2015; Blanch et al., 2012). By providing global coverage and the ability to ensure impeccable positioning solutions, global navigation satellite systems (GNSS) and thus all satellite systems currently operating for positioning and navigation can provide an essential foundation for contemporary and future, complex navigation concepts (Hegarty \& Chatre, 2008; Jin et al., 2011).

The increased private usage of these systems - for example, the use of mobile devices with localization functions - makes it clear that GNSS as a leading provider of geolocation is a technology that has moved into many applications of daily life and is crucial for the functioning of modern societies (Dow et al., 2009; European GNSS Agency, 2021). The high relevance of the subject is often based on the advantages of GNSS technology: it is a freely accessible system that, in addition to the oftenemphasized time, position, or even navigation-related aspects, is also capable of providing information about the characteristics of any surface from which a signal has been reflected (see Jaduszliwer \& Camparo, 2021). In conjunction with other possible application areas, including machine automation and surveying, this mapping leads to relevance in critical infrastructures, which are fundamental for the maintenance of societal functions, that cannot be neglected either (Aradau, 2010; Falletti et al., 2018; Thombre et al., 2018).

These publicly accessible facilities or installations are the main subjects of numerous debates in security research and, especially in the context of GNSS technology, highlight a need for improved robustness, accuracy, reliability, availability, and continuity of the technology. For example, numerous authors point out that the use of GNSS technology is often taken for granted and that both private users and operators of critical infrastructure are frequently unaware of the vulnerability of the systems (Ruegamer \& Kowalewski, 2015; Thombre et al., 2018). However, due to the interference of GNSS signals by specific jamming signals or other, primarily electronic systems, the applicability of GNSS in safety-critical contexts is fundamentally limited (Morong et al., 2019).

In this context, researchers emphasize - due to the fundamentally weak signals of the GNSS satellite - that it is necessary to ensure no disturbances in the GNSS frequency bands (Pullen \& Gao, 2012). However, since specific incidents repeatedly occur, which can be attributed to the use of jammers, it is necessary to address this issue in a particular way from a public safety perspective (Gao et al., 2016; Ruegamer \& Kowalewski, 2015). This necessity is mainly due to the fact that GNSS receivers are easily influenced and manipulated by the occurrence of various disturbances (interferences; e.g. Seo et al., 2015). Consequently, various interference events represent threats that must be taken seriously, require more precise classification, and must be brought to the attention of all users. 
The interference events defined in the context of GNSS issues are often divided into unintentional and intentional interferences (Lichtman et al., 2016). Within this paper, the focus is put on intentional interference, which is a criminal act and cyber threat. Examples for intentional interference are jamming and spoofing: jamming is the emission of a usually strong noise-like interfering signal to prevent a GNSS receiver from receiving and tracking the satellite signals and calculating its geo-location. Jamming is a denial-of-service attack and leads to a lack of position solutions or a decrease in position quality/accuracy. Broadcasting authentic but manipulated GNSSlike signals as a targeted attack to control users' computed position and timing solutions is referred to as spoofing (e.g., Gai et al., 2017; White et al., 1998). The group of structures affected by possible interference includes critical infrastructures and civilian areas such as central network operators that use GNSS technology too, for example, synchronize time networks (Broumandan et al., 2016).

Overall, numerous authors point to the devastating effects of various disruptive attacks in the context of criminal activity (e.g., Bhatti \& Humphreys, 2017; Psiaki \& Humphreys, 2016). Based on these findings and the already highlighted rapid increase in GNSS use in safety-critical applications, among others, the need for (a) designing the most comprehensive GNSS interference monitoring system possible, (b) investigating the potential impact of any interference, and (c) surveying the general awareness of the described problem from the public is apparent.

The issue now described is the starting point of the GIREKO project, which has been funded by the Austrian security research program KIRAS of the Federal Ministry of Agriculture, Regions and Tourism (BMLRT; see FFG KIRAS, 2018): In addition to the development of various technical solutions for the detection of possible interference events, from a socio-psychological point of view, an initial representation of the populations' state of knowledge or awareness of the GNSS as well as the GNSS interference topic should be made possible. Furthermore, the usage behavior, as well as possible prevailing feelings of dependency concerning the GNSS technology, should also be considered.

Thus, the present experimental study represents a first attempt to create awareness of the GNSS concept and GNSS interference with a focus on jamming and spoofing in the course of developing engineered solutions. In addition, an attempt is made to uncover the specific aspects relevant to the use of GNSS technology. Besides the individual GNSS usage, the GNSS acceptance, the self-assessed sense of orientation, the personal relevance for basic psychological needs, and a selection of often emphasized influencing factors concerning the acceptance and use of diverse technologies will be taken into account.

We note that the main focus of the present study was the aspect of GNSS navigation, as this can be considered the most apparent and relevant application for the public. 


\section{Method}

\section{Participants}

The dataset underlying further analyses includes data from 122 respondents (53 male, 66 female, three diverse) with a mean age of 34 years $(M=33.64, S D=13.99)$. Descriptive data on the professional background and highest completed educational level of the sample are presented in Table 1.

The sample attempted to recruit individuals of a wide range of walks of life to provide the most comprehensive picture of private GNSS users. Therefore, no specific exclusion criteria were defined besides age of majority and a linguistic understanding of the instructions.

\section{Table 1}

Distribution of the sample according to the highest level of education completed and the occupational situation prevailing at the time of the study

\begin{tabular}{lll}
\hline Level of Education & $N$ & $\%$ \\
No Degree & 0 & $0.0 \%$ \\
Compulsory School/Secondary School & 21 & $17.2 \%$ \\
Matura/Abitur/A-Level/High School Diploma & 41 & $33.6 \%$ \\
University Degree & 54 & $44.3 \%$ \\
Doctorate & 6 & $4.9 \%$ \\
\hline Current Employment & & \\
Student & $N$ & $\%$ \\
Worker & 28 & $23.0 \%$ \\
Employee & 10 & $8.2 \%$ \\
without management responsibility & 30 & $24.6 \%$ \\
Employee & & \\
with management responsibility & 30 & $24.6 \%$ \\
Entrepreneur & 30 & \\
Civil Servant & & $2.5 \%$ \\
Self-Employed Workers & 3 & $1.6 \%$ \\
Currently no Employment & $3.7 \%$ \\
Retired Person, Pensioner & 2 & $4.9 \%$ \\
\hline
\end{tabular}

\section{Research Material}

The test battery used in the online research study contained, in addition to a validated scale (described in more detail below), numerous specially created items and short 
information texts which provided the test subjects with a short explanation of the constructs in focus after the query of the currently prevailing level of knowledge. These short explanations were intended to ensure that valid statements were obtained concerning the factors queried and possibly directly related to GNSS use and the accompanying feeling of dependence.

In addition to a sociodemographic questionnaire, which recorded the personal data of age, gender, nationality, the level of education completed, and type of current employment while keeping anonymity intact, the subjects were also asked to provide information on various aspects of mobility. For example, they were asked to indicate how many steps and distances they walked on average per day. These statements were also asked for the corresponding habits concerning car and bicycle usage, if relevant to the individual.

Since one's own ability to orient and position oneself in space is a potential correlate in wayfinding performance (and thus probably also an important variable concerning the experience of dependency and usage behavior of GNSS devices; see Hund \& Nazarczuk, 2009), participants were presented with the Santa Barbara Sense-ofDirection Scale (SBSDS) by Hegarty et al. (2002). The scale consists of a total of 15 items, which are phrased as specific statements about a person's orientation behavior (e.g., "I am very good at giving directions" (positively phrased) or "I get lost very easily in a new city" (negatively phrased)). The items were to be scored on a seven-point Likert scale (from 1: "strongly agree" to 7: "strongly disagree"). A higher average score, which is calculated from the respondents' given ratings and can assume a value of one to seven as a total score, represents an orientation perceived as better.

The test battery also included items that asked about the perceived importance of specific psychological needs. We aimed to obtain a holistic picture of the aspects that are important to the respondents and to be able to conclude the possibly underestimated risks in connection with the intentional influences of GNSS technology. For this purpose, the subjects were asked to indicate on a 4-point scale (from 1: "Unimportant" to 4: "Important") how relevant the listed aspects (e.g., family, independence, justice) are to their personal lives. During the construction of the questionnaire, the aspects of privacy, self-determination, security, and technology were focused on, as these could be relevant in the context of the primary topic.

According to the Unified Theory of Acceptance and Use of Technology (UTAUT; Venkatesh et al., 2003), specific items generated constituted another part of the survey. Through the theory, which was initially designed to capture the acceptance and use of novel technological solutions, we captured the acceptance and use of various GNSS devices in the present study, allowing us to gain a first impression of the relevance of the contextual factors defined by Venkatesh et al. (2003), namely Performance Expectancy, Effort Expectancy, Social Influence, and Facilitating Conditions, on actual reported GNSS usage. By including the broader influencing 
factors of Hedonistic Motivation and Habits of Usage, these could also be included in considerations with the potential moderating variables of Gender, Age, and Voluntariness of Use (Venkatesh et al., 2012). In the present study, to capture the factors as mentioned above, subjects were asked to rate specific statements (e.g., "I like to use devices that use GNSS" or "People I care about would advise me to use devices that use GNSS") in terms of their agreement on a five-point Likert scale (from 0: "Strongly disagree" to 4: "Strongly agree"). The total score of the described factors is obtained by calculating the mean value over the assigned items.

Concerning the constructs in focus, the subjects were asked to state whether they were familiar with the terms GNSS, jamming, and spoofing and whether they knew what they meant. In order to capture dependency as well as usage behavior of GNSS devices, respondents were asked to indicate on a four- or five-point Likert scale how dependent they feel on GNSS technology (from 0: "I do not feel dependent on the technology at all" to 3: "I feel dependent on the technology described and often would not be able to find my way without it") and how often they use it (from 0: "I do not use the technology" to 4: "I always use the technology"). Finally, the test battery was completed by specific items asking subjects to indicate how they rated their overall knowledge of GNSS as well as GNSS interference on a 10-point scale (from 1: "Very little knowledge" to 10: "Very much knowledge").

\section{Procedure}

Participants were recruited via various social media platforms as well as personal contacts of the investigators. The survey took place online via a specific survey tool.

To create a standardized setting, participants were asked to complete the questionnaires in a quiet environment on an electronic device of their choice. After the standardized general instructions, subjects were asked to provide informed consent. The study participants were then presented first with the demographic questionnaire, the query on the level of knowledge and understanding of GNSS technology (followed by a brief informational text on the construct), the specific use or dependency scales, and the questions on various mobility aspects. The described items were followed by the items designed according to the UTAUT, the SBSDS, and assessing the personal relevance of psychological aspects to the subjects' personal lives. Before presenting another short informational text, the level of knowledge about the GNSS interference events jamming and spoofing was asked, and finally, the scale for the final overall assessment was given.

The test battery took about 12 to 15 minutes to complete.

\section{Statistical Design}

Various correlation and moderation analyses as well as a multiple linear regression were performed to uncover potential relationships and interactions in the context of 
individual GNSS use. Further exploratory research on the general population's level of knowledge and experienced dependence on GNSS technology was done.

\section{Results}

Data were tested using IBM SPSS 25.0.0.1 with an $\alpha$ of .05 for all statistical tests. Before the actual calculation steps, the variables in focus were checked for normal distribution, relying on the central limit theorem despite any violations of this due to the sufficiently large sample ( $N>30$; see Bühner \& Ziegler, 2009).

\section{GNSS, Jamming, and Spoofing}

Of the 122 subjects, $72(59.0 \%)$ reported not knowing what is meant by the term GNSS. $15(12.3 \%)$ were uncertain. A similar picture emerged concerning the level of knowledge or awareness of the specific GNSS interference events: 66 (54.1\%) and 62 $(50.8 \%)$ subjects reported that they were not familiar with the terms jamming and spoofing, respectively (see Table 2).

\section{Table 2}

Level of knowledge: GNSS, Jamming, and Spoofing

\begin{tabular}{lll}
\hline $\begin{array}{l}\text { Are you familiar with the term Jamming? } \\
\text { Do you know what that means? }\end{array}$ & N \\
Yes & 22 & $18.0 \%$ \\
No & 66 & $54.1 \%$ \\
Unsure & 15 & $12.3 \%$ \\
Missing & 19 & $15.6 \%$ \\
\hline Are you familiar with the term Spoofing? & & \\
Do you know what that means? & $N$ & \\
Yes & & \\
No & & $18.9 \%$ \\
Unsure & 23 & $50.8 \%$ \\
Missing & 62 & $14.8 \%$ \\
& 18 & $15.6 \%$ \\
\hline
\end{tabular}

In terms of the dependency and usage profile, a total of $60.7 \%$ of participants reported using GNSS technology often or always. $70.5 \%$ of subjects reported having to invest significantly more or being unable to navigate without the technology. In terms of the overall assessment, subjects reported low overall knowledge of both GNSS technology $(M=3.74, S D=2.00$; Range: $1-10)$ and various GNSS interference events $(M=4.12, S D$ = 2.25; Range: 1-10). 


\section{Aspects of mobility}

Regarding the subjects' aspects of mobility, the respondents reported to cover an average of 7236 steps $(S D=3884)$ daily as well as to travel $17.84 \mathrm{~km}(S D=24.09)$ by car and $3.34 \mathrm{~km}(S D=7.30)$ by bicycle daily. In terms of the number of distances or trips made by each mode of transportation, subjects reported an average of 5.54 (SD $=5.59)$ daily trips by foot, $1.79(S D=1.86)$ daily trips by car, and $0.50(S D=0.93)$ daily trips by bicycle.

It must be noted that the mentioned average values of mobility by bicycle and by car were only calculated for those who use the specific way of transport. Further analyses, which focus on the reported aspects, also only included the active users.

\section{Correlative Effects}

To uncover possible correlations and interactions with the variables in focus around the topics of GNSS or GNSS interference, numerous Pearson correlations were performed.

Significant correlations were found between the role as GNSS receiver (use of GNSS technology) and the personal relevance of self-determination $(r(101)=.203, p=$ $.041)$, the variable Effort Expectation of UTAUT $(r(112)=.231, p=.014)$, the UTAUTvariable Hedonistic Motivation $(r(111)=.332, p<.001)$, the UTAUT-variable Habits of Using $(r(112)=.266, p=.004)$, the number of steps traveled daily $(r(94)=.275, p=$ $.007)$, and the number of kilometers traveled daily by bicycle $(r(100)=-.257, p=$ .010). Thus, higher scores in these variables (i.e., self-determination experienced as more relevant, higher effort expectancy, higher hedonistic motivation, and more vigorous habits in the context of GNSS technology, and higher number of steps traveled daily) are associated with more frequent GNSS usage. In addition, the results indicate that the higher the number of kilometers traveled daily by bicycle, the less frequently individuals use GNSS technology.

Another relationship became evident between the reported GNSS dependence and the total SBSDS score $(r(112)=-.219, p=.020)$ : The higher the self-assessed orientation skills, the lower the dependence on GNSS.

Concerning the overall assessment of the GNSS issue, a significant correlation was found with the daily steps taken $(r(91)=.215, p=.040)$. Thus, individuals with a higher daily number of steps estimated their overall prevailing knowledge of GNSS higher than individuals who took fewer steps. In contrast, the overall assessment of the GNSS interference issue correlated with the effort expectancy subscale of the use or acceptance items developed on GNSS devices (UTAUT; $r(103)=.194, p=.048$ ). Further results of the Pearson correlations are shown in Table 3. 


\section{Table 3}

Pearson correlations on the specific GNSS variables (GNSS use or dependence), the relevance of specific psychological needs, the overall assessment on GNSS and GNSS interference, the SBSDS, the subscales of the UTAUT, and the specific aspects of mobility

\begin{tabular}{|c|c|c|c|c|c|c|c|c|c|c|c|c|c|c|c|c|c|c|c|c|c|}
\hline & 1. & 2. & 3. & 4. & 5. & 6. & 7. & 8. & 9. & 10. & 11. & 12. & 13. & 14. & 15. & 16. & 17. & 18. & 19. & 20. & 21. \\
\hline 1. GNSSlunge: & & & & & & & & & & & & & & & & & & & & & \\
\hline 2. GNSSOependenty & .069 & - & & & & & & & & & & & & & & & & & & & \\
\hline 3. Privacy & .046 & .123 & - & & & & & & & & & & & & & & & & & & \\
\hline $\begin{array}{l}\text { 4. Self- } \\
\text { Determination }\end{array}$ & $.203^{\circ}$ & .040 & .077 & - & & & & & & & & & & & & & & & & & \\
\hline 5. Safety & -012 & .086 & $.200^{\circ}$ & .122 & & & & & & & & & & & & & & & & & \\
\hline 6. Technology & .183 & $\begin{array}{l}-147 \\
\end{array}$ & .040 & .311 & .148 & . & & & & & & & & & & & & & & & \\
\hline 7. GNSSknowlogere & .126 & -088 & .008 & .049 & .006 & .116 & $\cdot$ & & & & & & & & & & & & & & \\
\hline $\begin{array}{l}8 . \\
\text { Interferencercomelsye }\end{array}$ & .161 & .088 & -009 & .019 & $.219^{*}$ & $.236^{*}$ & .130 & - & & & & & & & & & & & & & \\
\hline 9. SBSDS & .003 & $219^{*}$ & -077 & .126 & -159 & -146 & .132 & .004 & - & & & & & & & & & & & & \\
\hline $\begin{array}{l}10 \\
\text { UTAUT Potom Eneset }\end{array}$ & .141 & .168 & .222 & .005 & -114 & -113 & -162 & .000 & $.312^{*}$ & - & & & & & & & & & & & \\
\hline 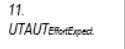 & $.231^{*}$ & .127 & .086 & .043 & .192 & .046 & .165 & $.194^{*}$ & -.076 & $.285^{*}$ & - & & & & & & & & & & \\
\hline 12. UTAUTScosast & .182 & .070 & .055 & .074 & .032 & .167 & .080 & .190 & $.213^{*}$ & $.336^{*}$ & .157 & & & & & & & & & & \\
\hline 13. UTAUTFrealicond & .131 & .077 & $.216^{*}$ & .186 & .008 & .172 & .063 & .117 & .001 & .166 & $.233^{\circ}$ & $.381^{\mathrm{tw+}}$ & - & & & & & & & & \\
\hline 14. UTAUTtrasliber & $.332^{\mathrm{met}}$ & .037 & .193 & .111 & .061 & .160 & .096 & .025 & .009 & .011 & $.411^{\mathrm{tw}}$ & $.253^{* \prime}$ & $.442^{2+0+}$ & - & & & & & & & \\
\hline 15. UTAUTHeobis & $.266^{*}$ & .001 & .087 & .102 & .070 & .107 & .083 & .163 & .088 & .129 & $.276^{*}$ & $.197^{\circ}$ & $.376^{\mathrm{tat}}$ & $.413^{\text {tw+ }}$ & - & & & & & & \\
\hline 16. Steps & $.275^{* *}$ & .079 & .002 & 1 & .078 & .016 & $.215^{*}$ & .071 & .022 & .157 & .047 & $.246^{*}$ & .093 & .141 & .128 & - & & & & & \\
\hline 17. Routessod & .041 & .061 & .049 & 190 & .029 & -.080 & .146 & .084 & .068 & .007 & .047 & .073 & .059 & -.020 & .076 & $.333^{* *}$ & - & & & & \\
\hline 18. Kilometerscor & .144 & .099 & .033 & .088 & .186 & .078 & .074 & .037 & $254^{* *}$ & -.001 & .116 & .069 & -.024 & .147 & .140 & .177 & .102 & - & & & \\
\hline 19. Routescor & .150 & -043 & .033 & .097 & .124 & .004 & .019 & .072 & -019 & -047 & .011 & .129 & .036 & .111 & .028 & .115 & .082 & $.613^{\mathrm{met}}$ & - & & \\
\hline 20. Kilometerss oprite & $.257^{*}$ & .142 & .186 & .028 & .196 & -130 & -106 & .018 & .024 & .126 & .093 & .059 & .070 & -122 & .016 & .001 & .083 & .082 & -148 & - & \\
\hline 21. Routessbrecte & -142 & .142 & $.219^{*}$ & .015 & .112 & -100 & .084 & .032 & .006 & .095 & -.060 & .095 & .175 & .007 & .144 & .002 & & -153 & 2320 & $.744^{\mathrm{tu}}$ & - \\
\hline
\end{tabular}

Note. ${ }^{*} p<.05 .{ }^{* *} p<.01 .{ }^{* * *} p<.001$.

\section{Moderating Effects}

We finally tried to uncover further interactions in the context of the focused variables, focusing on the use of GNSS devices using various moderation analyses based on the assumptions of UTAUT. When considering all UTAUT factors and the moderator variables defined by Venkatesh et al. (2012), significant interaction effects concerning GNSS use and the specific moderators emerged only in the analyses of performance expectancy, social influence, and habits of use. Due to the numerous UTAUT factors included in the analyses and the only predominant relevance of significant results, only the significant moderation effects now indicated will be reported below.

The PROCESS macro version 3.5 by Andrew Hayes (Hayes, 2017) was used for the tests to determine the exact effect of the identified interaction effects of the overall models. For an improved interpretation of the results, both the independent and moderator variables were centered in the course of the interaction effects, i.e., a 
middle point of the average values was constructed and the variables were considered in terms of a low as well as a high expression around this constructed point.

Thus, in the area of the UTAUT factor performance expectancy, only the expression of a person's age was found to affect the relationship between performance expectancy of a GNSS-enabled device and the use of such a device (overall model: $R^{2}=0.1154$, $F(3,80)=3.48, p=.020$, interaction effect: $\Delta R^{2}=0.0952, F(1,80)=8.61, p=.004$, $95 \% \mathrm{CI}[-0.043,-0.003])$. The effects indicate that only at a young age $(-1 S D)$ there is a significant positive relationship between a person's performance expectancy concerning GNSS technology and their use of it $(b=0.405, S E b=0.155, t=2.61, p=$ $.011,95 \% \mathrm{CI}[0.096,0.713])$. In the case of the age identified as average in the present study (M: $b=0.108, S E b=0.137, t=0.79, p=.430,95 \% C I[-0.1636,0.3803])$ and an age defined as higher in this context $(+1 S D: b=-0.188, S E b=0.184, t=-1.02, p=.309$, $95 \% \mathrm{CI}[-0.5537,0.1776])$, no significant effects of age on the considered relationship between performance expectancy and GNSS use were found.

Beyond this interaction effect, there was a significant interaction between the age of GNSS users and the social influence on technology (overall model: $R^{2}=0.1417$, $F(3,109)=6.00, p=.001$; interaction effect: $\Delta R^{2}=0.08, F(1,109)=10.194, p=.002$, $95 \% \mathrm{CI}[-0.033,-0.004])$. Thus, a person's age also influences the effect of social influence in the context of GNSS device use. The effects indicate that only at a younger age (-1SD) there is a significant relationship between the social influence in GNSS technology acceptance and use $(b=0.468, S E b=0.127, t=3.68, p<.001$, $95 \% \mathrm{CI}[0.2158,0.7193])$. If individuals were in the same age range as the sample's average age ( $M: b=0.178, S E b=0.109, t=1.64, p=.104,95 \% \mathrm{CI}[-0.0373,0.3926])$ or older (+1SD: $b=-0.112, S E b=0.155, t=-0.73, p=.469,95 \% \operatorname{CI}[-0.419,0.194])$, no significant effects of age on the considered relationship between the social influence in the context of GNSS technologies and GNSS use could be found.

Finally, the results indicated a moderator function of voluntariness in the use of any systems concerning the relationship between the habit of use and actual GNSS use (overall model: $R^{2}=0.1387, F(3,107)=5.74, p=.001$; interaction: $\Delta R^{2}=0.04, F(1,107)$ $=4.91, p=.029,95 \% \mathrm{CI}[-0.252,0.031])$. A closer look at the follow-up tests revealed that only when individuals indicated a low $(-1 S D: b=0.36, \mathrm{SEb}=0.11, t=3.27, p=$ $.001,95 \% \mathrm{CI}[0.142,0.577])$ or mean $(M: b=0.18, S E b=0.09, t=-2.12, p=.036$, $95 \% \mathrm{CI}[0.012,0.356])$ voluntariness in using GNSS technology, a significant positive relationship between habits of use in using GNSS devices and actual use was found. The relationship between habits of use and GNSS use is, thus, significant only for individuals who have a low or medium level of voluntariness in use. On the other hand, with a high degree of voluntariness, there is no significant effect of voluntariness of use on the considered relationship ( $+1 S D: b=0.01, S E b=0.12, t=$ $0.07, p=.948,95 \% \mathrm{CI}[-0.239,0.255]$; see Figure 1 ). 


\section{Figure 1}

Moderation analysis to predict GNSS use by habits and voluntariness in using the systems

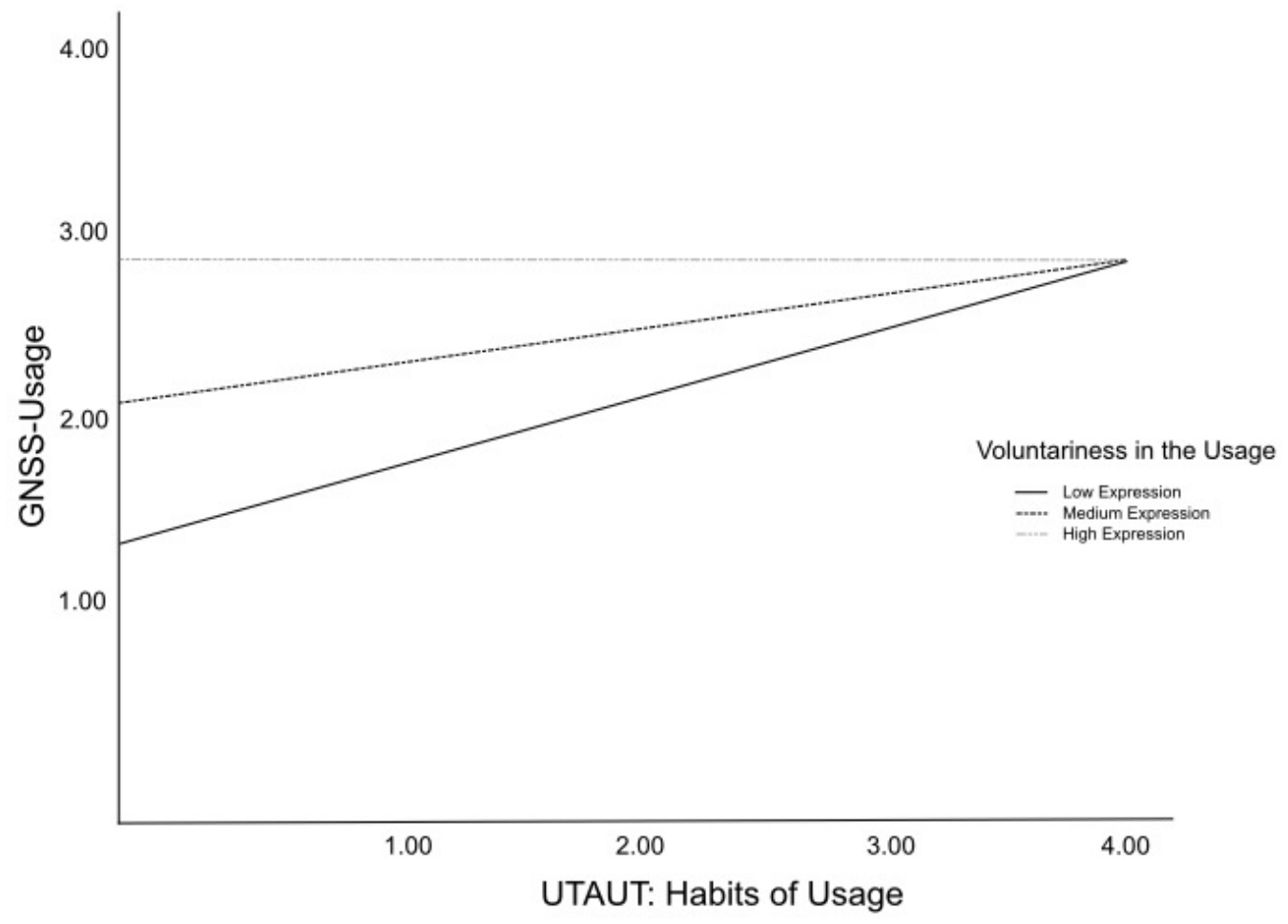

Note. Overall model: $R^{2}=0.1387, F(3,107)=5.74, p=.001$; Interaction: $\Delta R^{2}=0.04, F(1,107)=4.91, p=.029,95 \% \operatorname{CI}[-0.252,0.031]$.

\section{Multiple linear Regression}

Multiple linear regression was used to identify a specific model based on the variables focused on in this study that could predict GNSS device use. The relationships uncovered for GNSS use served as the basis for these considerations.

The model based on the revealed correlations and also moderations, which includes the predictors' number of steps per day, the average number of kilometers traveled daily by bicycle, the relevance of self-determination, the expectation of effort, hedonistic motivation, habits in using GNSS, and voluntariness in using any systems, proves to be satisfactory with a high variance explanation $\left(R^{2}=.404\right)$ and can thus be used to predict GNSS use $(F(7,68)=6.57, p<.001)$. 
However, when taking a closer look at the specific effects, only the variables number of steps traveled per day $(\beta=.325, p=.002)$, the number of kilometers traveled by bicycle $(\beta=-.279, p=.005)$, the relevance of the aspect self-determination $(\beta=.222$, $p=.026)$, and hedonistic motivation $(\beta=.250, p=.036)$ make a significant contribution to predicting GNSS use. Through the results, GNSS use increases with the number of steps traveled per day, self-assessed relevance of self-determination, and stated hedonistic motivation related to GNSS use. Concerning the number of kilometers traveled per day by bicycle, an adverse effect can be reported: If the number of kilometers traveled increases, GNSS use seems to decrease.

A model designed following these considerations, including only the factors identified as significant $\left(R^{2}=.392, F(4,73)=11.77, p<.001\right)$, is also able to predict GNSS use significantly and must be considered the best model for predicting GNSS use due to the exclusive inclusion of significant variables (see Figure 2).

\section{Figure 2}

Prediction model for GNSS usage

Steps taken per day and number of kilometers traveled per day (bicycle)

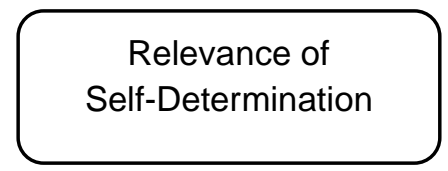

Extended Factor of the Unified Theory of Acceptance and Use of Technology

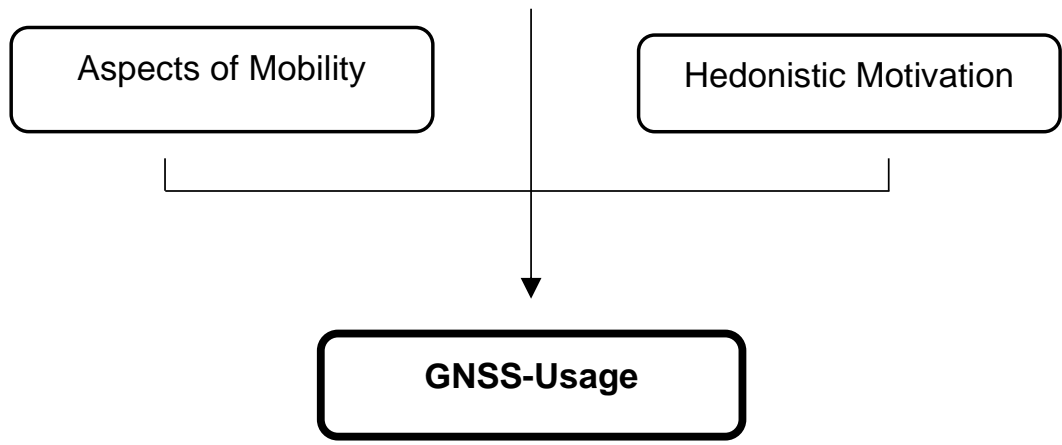

\section{Discussion}

The present study examined the interactions and interdependencies of GNSS-enabled device use and focused on the level of knowledge or awareness of the subject of GNSS and the GNSS interference events of jamming and spoofing.

In addition to a deficient level of knowledge about the described constructs and a significant correlation between GNSS use and personal relevance of selfdetermination, essential correlates of the focused variable with the specific UTAUT 
factors of effort expectancy, hedonistic motivation, and habits of using any devices also emerged. In addition, it became clear that specific aspects of personal mobility (steps taken daily, kilometers cycled daily) were also correlated with GNSS use.

Additional correlations in the context of the GNSS construct or the specific GNSS interference events were evident between reported GNSS dependence and selfassessed orientation skills. The overall assessment of the GNSS issue correlated with steps traveled daily and the overall assessment on the GNSS interference issue correlated with the UTAUT factor effort expectancy.

As a result of further analysis, it became clear that a person's age influences both the relationship between GNSS-enabled devices' performance expectancy and use and the relationship between social influence in GNSS use and actual use. The aspect of voluntariness in using GNSS-enabled devices was also identified as a moderator of the relationship between habits of use and actual GNSS use.

Finally, it became clear that the number of steps taken daily can predict the use of GNSS-enabled devices, the number of kilometers cycled daily, the personal relevance of self-determination, and a person's hedonistic motivation.

\section{Positioning in the context of previous literature}

All studies which can be classified mainly in the field of safety research indicate that human navigation using different systems is a very complex interaction, requiring detailed yet comprehensive individual data to capture the basic psychological mechanisms (see Irmischer \& Clarke, 2018; Maciuk \& Rudyk, 2020; Miyazawa et al., 2020). The present study represents one of the few existing attempts to investigate the interrelationships and interactions related to satellite positioning systems for human navigation in a social science-psychological context. Thus, concerning previous literature in the GNSS context, it must be pointed out that these often dealt with a technical perspective or only focused on particular aspects of human nature (e.g., perception and cognition, Sun et al., 2016) in the course of GNSS navigation.

The present study extends previous literature. It has been shown that specific skills or aspects of an individual are relevant in GNSS use. Because of the increasing linkage of various telecommunication technologies with GNSS technology, the psychological perspective should also assume a fundamental role in future research on spacerelated technologies.

\section{Limitations of the study and implications for future research}

The present study is characterized by several limitations, some of which lead to a limited significance of the results and need to be considered in future studies.

An essential part of the test battery used is the UTAUT in its extended form (Venkatesh et al., 2012). It must be noted that despite the consideration of numerous factors, the completeness of the model is not given in the version used. For example, 
the factors of experience and behavioral intention concerning the use of various technologies and the variable of price value were not included. Although the factors were deliberately excluded due to practical considerations (often not possible or meaningful item generation), future studies should address these aspects and implement them accordingly in the GNSS context.

In the context of the presented UTAUT items, it should also be noted that only up to four items were assigned to each of the corresponding factors. Future approaches should also consider this aspect and provide a more significant number of corresponding items in terms of the desired meaningfulness of the scales.

Concerning the aspects of mobility surveyed in the present study, it must be mentioned that the estimation of the steps or even kilometers traveled daily or the corresponding numbers of distances could be complex for the respondents. The descriptive data of these items clearly show the variability in the respondents' statements due to a partly very high scatter range and reinforce the suspicion of uncertainty in answering the items. In future studies, it should be explained more specifically and possibly with the help of an example how the respective information can be estimated in an improved way.

Overall, it must also be noted that the scales and items used throughout are self-report instruments. It is assumed that data based on self-assessment are generally subject to bias and other psychosocial factors, which may have misrepresented the resulting data and thus skewed the results. Objective data collection concepts, and thus, for example, recourse to the pedometers often built into mobile devices, could provide more reliable information, especially in the area of daily steps taken and should be considered in future studies.

The present study was a first attempt to uncover the state of knowledge or awareness of the GNSS (interference) issue and relate the associated aspects (e.g., GNSS dependency, GNSS use, overall assessment of the state of knowledge on specific topics) to specific variables. Future studies should capture these variables in focus more precisely and thus, for example, supplement the frequency statements to be selected in the context of GNSS use (e.g., "Occasionally," "Often") with appropriate objective comparison values (e.g., "at least once a month," "at least twice a week"). In this way, more specific statements can be made in further consideration of the constructs.

\section{Implications}

Despite the limitations mentioned above, the present study contributes significantly to an improved understanding of GNSS use and the aspects relevant in this context. It provides essential insights for psychological-social science research in security research, which focuses on the GNSS technology and has been investigated mainly by technical disciplines (e.g., software development; Barbarella et al., 2009). 
The present findings provide important insight into the general level of knowledge of private GNSS users and respond to a prevailing desire for increased attention to population-based aspects in the area of human-technology interface and interaction. In addition, the results can be used to prepare empirical exercises and as a basis for further approaches (for example, in the course of various educational campaigns). It is thus possible to emphasize the high relevance of the public perspective in the context of GNSS technology research, which must also be taken into account in future research.

\section{Conclusion}

The increasing use of GNSS-enabled devices and the threat situations that are also increasing in this context require research into specific correlations and interactions in the development of technical solutions and the socio-scientific-psychological field.

The present study investigated the use of GNSS-enabled devices in a correlative, moderating, and linear manner and was able to draw attention to the relevance of the age of corresponding users and the importance of the experienced voluntariness in the use of corresponding devices. The psychological need for self-determination, specific aspects of mobility, and the variables of effort expectation, hedonistic motivation, and habits of use often reported in the context of technology use and acceptance are also highly relevant in GNSS technology.

Due to the ever-increasing focus on security-related aspects and the need to ensure protection against all interference events, which is often emphasized in the GNSS construct, an in-depth examination of the interrelationships and interactions of technology use is indispensable. In order to act in terms of preventing crisis events, it is essential to explore aspects of GNSS dependency further and use them in addition to clarifying the constructs focused on in this study.

\section{Acknowledgments}

The GIREKO project (GNSS Interference Map Austria), in which we wrote this paper, has been funded by the Austrian security research program KIRAS of the Federal Ministry of Agriculture, Regions and Tourism (BMLRT). The authors would like to thank the organizers of this program and especially the consortium of the project for theoretical support and conception and emphasize their relevance for the development of the paper.

FFG-Nr. 873435, project or cooperation partners: JOANNEUM RESEARCH Forschungsgesellschaft $\mathrm{mbH}$ (Forschungsgruppe Weltraumtechnik und Kommunikationstechnologien, Institut DIGITAL), IGASPIN GmbH, Bundesministerium für Landesverteidigung, and ERC Experience Research \& Consulting. 


\section{References}

[1] Aradau, C. (2010). Security that matters: Critical infrastructure and objects of protection. Security dialogue, 41(5),491-514. https://doi.org/10.1177/0967010610382687

[2] Barbarella, M., Cenni, N., Gandolfi, S., Ricucci, L., \& Zanutta, A. (2009). Technical and scientific aspects derived by the processing of GNSS networks using different approaches and software. Proceedings of the 22nd International Technical Meeting of the Satellite Division of The Institute of Navigation (ION GNSS 2009), 2677-2688.

[3] Bhatti, J., \& Humphreys, T. E. (2017). Hostile control of ships via false GPS signals: Demonstration and detection. NAVIGATION, Journal of the Institute of Navigation, 64(1), 51-66. https://doi.org/10.1002/navi.183

[4] Blanch, J., Walter, T., \& Enge, P. (2012). Satellite navigation for aviation in 2025. Proceedings of the IEEE, 100 (Special Centennial Issue), 1821-1830. https://doi.org/10.1109/JPROC.2012.2190154

[5] Broumandan, A., Jafarnia-Jahromi, A., Daneshmand, S., \& Lachapelle, G. (2016). Overview of spatial processing approaches for GNSS structural interference detection and mitigation. Proceedings of the IEEE, 104(6), 12461257.

[6] Bühner, M., \& Ziegler, M. (2009). Statistik für Psychologen und Sozialwissenschaftler. Pearson Deutschland GmbH.

[7] Dovis, F. (Ed.). (2015). GNSS interference threats and countermeasures. Artech House.

[8] Dow, J. M., Neilan, R. E., \& Rizos, C. (2009). The international GNSS service in a changing landscape of global navigation satellite systems. Journal of geodesy, 83(3), 191-198. https://doi.org/10.1007/s00190-008-0300-3

[9] European GNSS Agency (GSA), "Market report issue 6," European Global Navigation Satellite Systems Agency. Retrieved July 20, 2021, from https://www.euspa.europa.eu/european-space/euspace-market/gnssmarket/gnss market-report

[10] Falletti, E., Margaria, D., Marucco, G., Motella, B., Nicola, M., \& Pini, M. (2018). Synchronization of critical infrastructures dependent upon GNSS: Current vulnerabilities and protection provided by new signals. IEEE Systems Journal, 13(3), 2118-2129. https://doi.org/10.1109/JSYST.2018.2883752

[11] FFG KIRAS (2018). GIREKO - GNSS Interferenz Karte Österreich. Retrieved August 02, 2021, from https://www.kiras.at/gefoerderteprojekte/detail/d/girekognssinterferenzkarte-oesterreich/

[12] Gai, K., Qiu, M., Ming, Z., Zhao, H., \& Qiu, L. (2017). Spoofing-jamming attack strategy using optimal power distributions in wireless smart grid 
networks. IEEE Transactions on Smart Grid, 8(5), 2431-2439. https://doi.org/10.1109/TSG.2017.2664043

[13] Gao, G. X., Sgammini, M., Lu, M., \& Kubo, N. (2016). Protecting GNSS receivers from jamming and interference. Proceedings of the IEEE, 104(6), 1327-1338. https://doi.org/10.1109/JPROC.2016.2525938

[14] Hayes, A. F. (2017). Introduction to mediation, moderation, and conditional process analysis: A regression-based approach. Guilford publications.

[15] Hegarty, C. J., \& Chatre, E. (2008). Evolution of the global navigation satellitesystem (gnss). Proceedings of the IEEE, 96(12), 1902-1917. https://doi.org/10.1109/JPROC.2008.2006090

[16] Hegarty, M., Richardson, A. E., Montello, D. R., Lovelace, K., \& Subbiah, I. (2002). Development of a self-report measure of environmental spatial ability. Intelligence, 30(5), 425-447. https://doi.org/10.1016/S01602896(02)00116-2

[17] Hund, A. M., \& Nazarczuk, S. N. (2009). The effects of sense of direction and training experience on wayfinding efficiency. Journal of Environmental Psychology, 29(1), 151-159. https://doi.org/10.1016/j.jenvp.2008.05.009

[18] Irmischer, I. J., \& Clarke, K. C. (2018). Measuring and modeling the speed of human navigation. Cartography and Geographic Information Science, 45(2), 177-186. https://doi.org/10.1080/15230406.2017.1292150

[19] Jaduszliwer, B., \& Camparo, J. (2021). Past, present and future of atomic clocks for GNSS. GPS Solutions, 25(1), 1-13. https://doi.org/10.1007/s10291-020-01059-x

[20] Jin, S., Feng, G. P., \& Gleason, S. (2011). Remote sensing using GNSS signals: Current status and future directions. Advances in space research, 47(10), 1645-1653. https://doi.org/10.1016/j.asr.2011.01.036

[21] Lichtman, M., Poston, J. D., Amuru, S., Shahriar, C., Clancy, T. C., Buehrer, R. M., \& Reed, J. H. (2016). A communication jamming taxonomy. IEEE Security \& Privacy, 14(1), 47-54. https://doi.org/10.1109/MSP.2016.13

[22] Maciuk, K. \& Rudyk, Y. (2020). Usage of the global navigation satellite systems in safety and protection issues. Scientific Journal of Silesian University of Technology, 109, 93-102. https://doi.org/10.20858/sjsutst.2020.109.9

[23] Miyazawa, S., Song, X., Jiang, R., Fan, Z., Shibasaki, R., \& Sato, T. (2020). CITYSCALE HUMAN MOBILITY PREDICTION MODEL BY INTEGRATING GNSS TRAJECTORIES AND SNS DATA USING LONG SHORT-TERM MEMORY. ISPRS Annals of Photogrammetry, Remote Sensing \& Spatial Information Sciences, 5(4), 8794. https://doi.org/10.5194/isprs-annals-V-4-2020-872020

[24] Morong, T., Puričer, P., \& Kovář, P. (2019). Study of the GNSS jamming in real environment. International Journal of Electronics and Telecommunications, 65(1), 65-70. https://doi.org/10.24425/ijet.2019.126284 
[25] Psiaki, M. L., \& Humphreys, T. E. (2016). GNSS spoofing and detection. Proceedings of the IEEE, 104(6), 1258-1270. https://doi.org/10.1109/JPROC.2016.2526658

[26] Pullen, S., \& Gao, G. X. (2012). GNSS jamming in the name of privacy: Potential threat to GPS aviation. Inside GNSS, 7(2), 34-43.

[27] Ruegamer, A., \& Kowalewski, D. (2015). Jamming and spoofing of gnss signals-an underestimated risk?!. Proc. Wisdom Ages Challenges Modern World, 3, 17-21.

[28] Seo, S. H., Lee, B. H., Im, S. H., \& Jee, G. I. (2015). Effect of spoofing on unmanned aerial vehicle using counterfeited GPS signal. Journal of Positioning, Navigation, and Timing, 4(2), 57-65. https://doi.org/10.11003/JPNT.2015.4.2.057

[29] Sun, Q., Xia, J., Nadarajah, N., Falkmer, T., Foster, J., \& Lee, H. (2016). Assessing drivers' visual-motor coordination using eye tracking, GNSS and GIS: a spatial turn in driving psychology. Journal of spatial science, 61(2), 299-316. https://doi.org/10.1080/14498596.2016.1149116

[30] Thombre, S., Bhuiyan, M. Z. H., Eliardsson, P., Gabrielsson, B., Pattinson, M., Dumville, M.,... \& Kuusniemi, H. (2018). GNSS threat monitoring and reporting: Past, present, and a proposed future. The Journal of Navigation, 71(3), 513-529. https://doi.org/10.1017/S0373463317000911

[31] Venkatesh, V., Morris, M. G., Davis, G. B., \& Davis, F. D. (2003). User acceptance of information technology: Toward a unified view. MIS quarterly, 27(3), 425-478. https://doi.org/10.2307/30036540

[32] Venkatesh, V., Thong, J. Y., \& Xu, X. (2012). Consumer acceptance and use of information technology: extending the unified theory of acceptance and use of technology. MIS quarterly, 36(1), 157-178. https://doi.org/10.2307/41410412

[33] White, N. A., Maybeck, P. S., \& DeVilbiss, S. L. (1998). MMAE detection of interference/jamming and spoofing in a DGPS-aided inertial system. Proceedings of the 11th International Technical Meeting of the Satellite Division of the Institute of Navigation (ION GPS 1998), 905-914. 